\title{
Untangling Noncommutativity with Operator Integrals
}

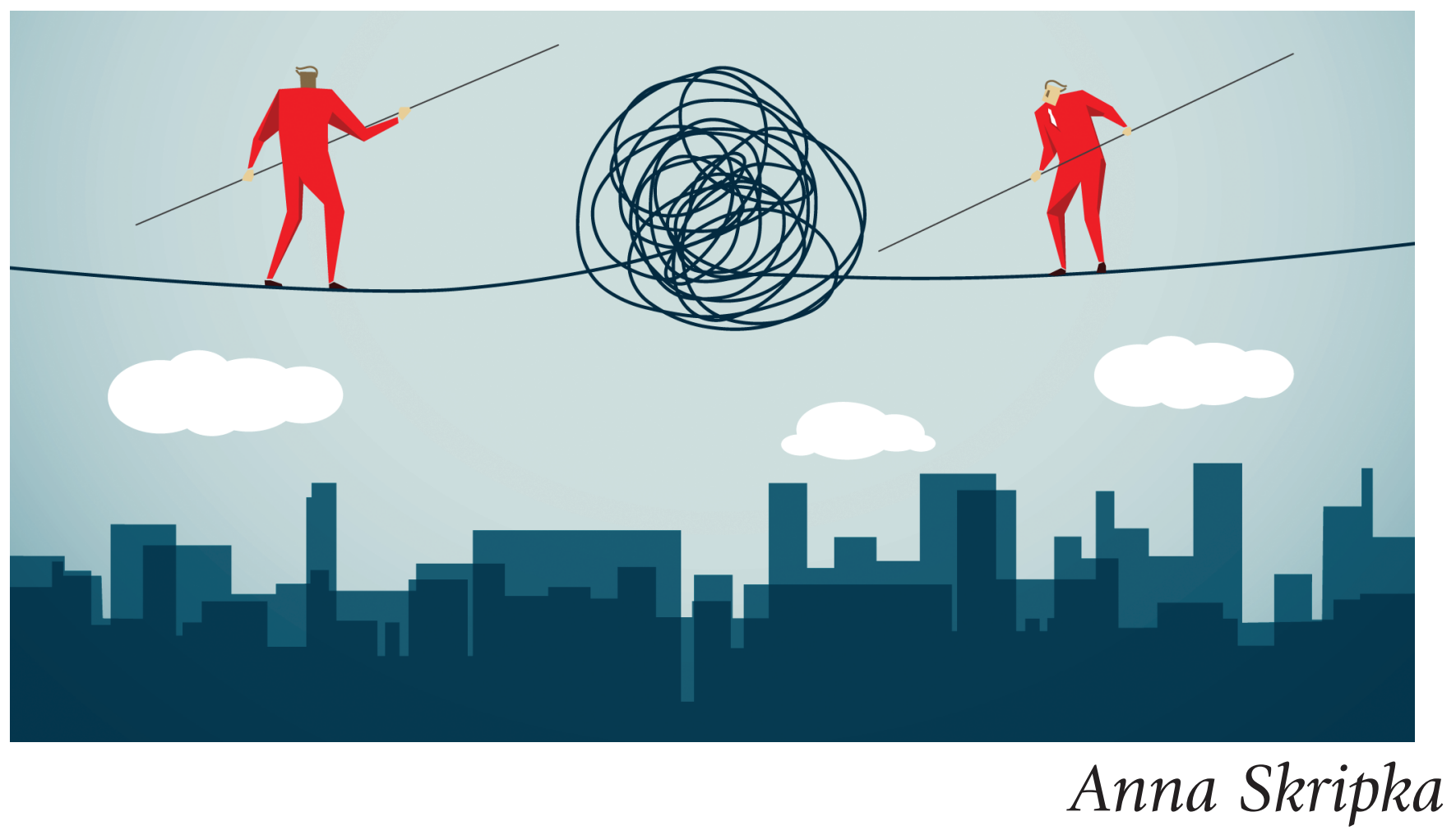

\section{Emergence of Operator Integration}

Operator integration (OI) is a collection of powerful methods and techniques that enable analysis of functions with noncommuting arguments. Such functions arise, in particular, in various problems of applied matrix analysis, mathematical physics, noncommutative geometry, and statistical estimation.

Single operator integrals are basic tools in the classical functional calculus. For instance, a self-adjoint operator $A$ densely defined in a separable Hilbert space admits the operator integral decomposition

$$
A=\int_{\mathbb{R}} \lambda d \varepsilon_{A}(\lambda)
$$

Anna Skripka is an associate professor of mathematics at the University of New Mexico. Her email address is skripka@math.unm. edu.

The author's work is partially supported by NSF CAREER grant DMS-1554456.

Communicated by Notices Associate Editor Daniela De Silva.

For permission to reprint this article, please contact:

reprint-permission@ams.org.

DOI: https://doi.org/10.1090/noti2008 where $\varepsilon_{A}$ is the projection-valued spectral measure of $A$, and the function of this operator $f(A)$ is given by the operator integral

$$
f(A)=\int_{\mathbb{R}} f(\lambda) d \varepsilon_{A}(\lambda)
$$

When $A$ is a finite matrix, the above integral degenerates to a finite sum

$$
f(A)=\sum_{k} f\left(\lambda_{k}\right) \mathcal{E}_{A}\left(\lambda_{k}\right) .
$$

This spectral integral (or sum) decomposition induces a straightforward estimate of an operator function in terms of the scalar function

$$
\|f(A)\| \leq\|f\|_{\infty} .
$$

In approximation problems one might need to estimate the difference $f(A)-f(B)$ of functions of self-adjoint operators $A$ and $B$ in terms of some norms of $A-B$ and $f$. When $A$ and $B$ are bounded and commuting, we can 
represent this difference as the integral

$$
\begin{aligned}
& f(A)-f(B) \\
& =\iint_{\mathbb{R}^{2}} \frac{f(\lambda)-f(\mu)}{\lambda-\mu} d \varepsilon_{A+i B}(\lambda, \mu)(A-B)
\end{aligned}
$$

with respect to the spectral measure of the normal operator $A+i B$. By submultiplicativity of the operator norm and properties of the spectral integral, we deduce the bound

$$
\|f(A)-f(B)\| \leq\|f\|_{\operatorname{Lip}(\mathbb{R})}\|A-B\| .
$$

Similar bounds for noncommuting $A$ and $B$ are deep results grounded on double or iterated operator integral decompositions

$$
\begin{aligned}
& f(A)-f(B) \\
& =\int_{\mathbb{R}} \int_{\mathbb{R}} \frac{f(\lambda)-f(\mu)}{\lambda-\mu} d \varepsilon_{A}(\lambda)(A-B) d \varepsilon_{B}(\mu) .
\end{aligned}
$$

The right-hand side above can be interpreted as the value of a linear transformation $T_{\varphi}^{A, B}$ on $A-B$, where $\varphi(\lambda, \mu)=$ $\frac{f(\lambda)-f(\mu)}{\lambda-\mu}$, and, thus,

$$
f(A)-f(B)=T_{\varphi}^{A, B}(A-B) .
$$

More generally, the OI approach reduces analysis of different noncommutative expressions to the analysis of a multilinear transformation $T_{\varphi}^{A_{1}, \ldots, A_{n+1}}$ acting on a Cartesian product of matrices or infinite-dimensional operators. The parameters $\varphi, A_{1}, \ldots, A_{n+1}$ are determined by the model in question. The values of the transformation $T_{\varphi}^{A_{1}, \ldots, A_{n+1}}$ are often decomposed into integrals with operator-valued integrands or measures. Properties of $T_{\varphi}^{A_{1}, \ldots, A_{n+1}}$ depend on the space where it acts, on the type of symbol $\varphi$, and sometimes on the spectral properties of the operators $A_{1}, \ldots, A_{n+1}$.

\section{Organization}

Our first acquaintance with multiple operator integration in this note will occur in the finite-dimensional setting since $T_{\varphi}^{A_{1}, \ldots, A_{n+1}}$ on tuples of matrices admits a finite sum representation. We will see a nonchronological summary of major results and glimpses of fundamental ideas along with questions approachable by the OI method.

Then we will become familiar with OI in the general setting of noncommutative $L^{p}$-spaces, where in addition to puzzles of noncommutativity one deals with convergence issues. We will touch upon several constructions known under the name "multiple operator integral," each one supplying a particular type of estimate for noncommutative expressions arising in different setups.

From the beginning of its development, the theory of operator integration has been motivated and guided by applications, and this synergy will be reflected in this note. We will demonstrate applicability of OI to questions arising in the study of smoothness properties of operator functions, spectral shift, spectral flow, quantum differentiability, and smoothness of noncommutative $L^{p}$. norms.

Technical details will be omitted, but an interested reader is invited to find them along with a continued discussion in the recent book [19]. Due to the restriction on the allowed number of references many important contributions in the field will not be cited here, but can be found in [19].

\section{Operator Integrals on Finite Matrices}

Methods of operator integration have been actively used and rediscovered in matrix analysis, often with many particular cases treated separately without appeal to a general theory. In this section we give an overview of general results supplied by the OI approach along with types of problems where they can be applied.

Linear case. Let $\ell_{d}^{2}$ denote the $d$-dimensional Hilbert space equipped with the Euclidean inner product and let $\mathcal{B}\left(\ell_{d}^{2}\right)$ denote the Banach space of linear operators (or $d \times d$ matrices) on $\ell_{d}^{2}$ equipped with the operator (spectral) norm. Let $A, B \in \mathcal{B}\left(\ell_{d}^{2}\right)$ be self-adjoint matrices, let $\left\{g_{j}\right\}_{j=1}^{d},\left\{h_{k}\right\}_{k=1}^{d}$ be complete systems of orthonormal eigenvectors, and let $\left\{\lambda_{j}\right\}_{j=1}^{d},\left\{\mu_{k}\right\}_{k=1}^{d}$ be sequences of the corresponding eigenvalues of $A$ and $B$, respectively. Let $P_{h}$ denote the orthogonal projection onto the vector $h \in \ell_{d}^{2}$ and let $\varphi: \mathbb{R}^{2} \rightarrow \mathbb{C}$ be a function.

The double operator integral constructed from the spectral data of $A, B$ and the symbol $\varphi$ is the bounded linear transformation

given by

$$
T_{\varphi}^{A, B}: \mathcal{B}\left(\ell_{d}^{2}\right) \rightarrow \mathcal{B}\left(\ell_{d}^{2}\right)
$$

$$
T_{\varphi}^{A, B}(X)=\sum_{j=1}^{d} \sum_{k=1}^{d} \varphi\left(\lambda_{j}, \mu_{k}\right) P_{g_{j}} X P_{h_{k}}
$$

for $X \in \mathcal{B}\left(\ell_{d}^{2}\right)$. In other words, $T_{\varphi}^{A, B}$ acts on $X$ as the entrywise multiplier of the matrix of $X$ in the bases $\left\{g_{j}\right\}_{j=1}^{d}$ and $\left\{h_{k}\right\}_{k=1}^{d}$ by the matrix $\left(\varphi\left(\lambda_{j}, \mu_{k}\right)\right)_{j, k=1}^{d}$ :

$$
\left(x_{j k}\right)_{j, k=1}^{d} \mapsto\left(\varphi\left(\lambda_{j}, \mu_{k}\right) x_{j k}\right)_{j, k=1}^{d} .
$$

Due to this interpretation, the transformation $T_{\varphi}^{A, B}$ is also called a Schur multiplier.

Given a differentiable function $f: \mathbb{R} \rightarrow \mathbb{C}$ and the matrix functions $f(A)$ and $f(B)$ defined by the functional calculus,

$$
f(A)=\sum_{j=1}^{d} f\left(\lambda_{j}\right) P_{g_{j}} \quad \text { and } \quad f(B)=\sum_{k=1}^{d} f\left(\mu_{k}\right) P_{h_{k}},
$$


we have the representation

$$
f(A)-f(B)=T_{f^{[1]}}^{A, B}(A-B) .
$$

Here $f^{[1]}$ is the divided difference of $f$ given by

$$
f^{[1]}(\lambda, \mu)= \begin{cases}\frac{f(\lambda)-f(\mu)}{\lambda-\mu} & \text { if } \lambda \neq \mu, \\ f^{\prime}(\lambda) & \text { if } \lambda=\mu .\end{cases}
$$

The representation (3) was derived by K. Löwner in 1934 in his work on characterization of matrix monotone functions by means of basic spectral theory. From (3) and the Schur multiplier property (2) of $T_{f^{[1]}}^{A, B}$ we conclude that if the matrix $\left(f^{[1]}\left(\lambda_{j}, \mu_{k}\right)\right)_{j, k=1}^{d}$ is positive definite, then the function $f$ is matrix monotone, that is, $A \leq B$ implies $f(A) \leq f(B)$.

The representation (3) along with (1) can be used to prove existence and the Schur multiplier property (2) of the matrix derivative

$$
\left.\frac{d}{d t} f(B+t(A-B))\right|_{t=0}=T_{f^{[1]}}^{B, B}(A-B) .
$$

The double operator integral also calculates the quasicommutator

$$
f(A) X-X f(B)=T_{f^{[1]}}^{A, B}(A X-X B) .
$$

The representations (3) - (5) indicate that increments and derivatives of operator functions as well as quasicommutators can be studied and estimated in a unified way in the double operator integration framework. We will consider a variety of such estimates in different norms.

Multilinear case. Multilinear operator integrals arise as natural extensions of double ones in higher-order perturbation problems.

Let $n$ be a natural number, let $A_{1}, \ldots, A_{n+1} \in \mathcal{B}\left(\ell_{d}^{2}\right)$ be self-adjoint matrices, let $\left\{\mathrm{g}_{i}^{(j)}\right\}_{i=1}^{d}$ be an orthonormal basis of eigenvectors, and let $\left\{\lambda_{i}^{(j)}\right\}_{i=1}^{d}$ be the corresponding sequence of eigenvalues of $A_{j}$ for $j=1, \ldots, n+1$. Let $\varphi: \mathbb{R}^{n+1} \rightarrow \mathbb{C}$ be a function.

The multilinear operator integral constructed from the spectral data of $A_{1}, \ldots, A_{n+1}$ and the symbol $\varphi$ is the bounded $n$-linear transformation

$$
T_{\varphi}^{A_{1}, \ldots, A_{n+1}}: \underbrace{\mathcal{B}\left(\ell_{d}^{2}\right) \times \cdots \times \mathcal{B}\left(\ell_{d}^{2}\right)}_{n \text { times }} \rightarrow \mathcal{B}\left(\ell_{d}^{2}\right)
$$

given by

$$
\begin{aligned}
& T_{\varphi}^{A_{1}, A_{2}, \ldots, A_{n+1}}\left(X_{1}, X_{2}, \ldots, X_{n}\right) \\
& =\sum_{r_{1}, r_{2}, \ldots, r_{n+1}=1}^{d} \varphi\left(\lambda_{r_{1}}^{(1)}, \lambda_{r_{2}}^{(2)}, \ldots, \lambda_{r_{n+1}}^{(n+1)}\right) \\
& \quad \cdot P_{g_{r_{1}}(1)} X_{1} P_{g_{r_{2}}(2)} X_{2} \cdots X_{n} P_{g_{r_{n+1}}(n+1)}
\end{aligned}
$$

for $X_{1}, X_{2}, \ldots, X_{n} \in \mathcal{B}\left(\ell_{d}^{2}\right)$. It is also called an $n$-linear Schur multiplier.

The transformation $T_{\varphi}^{A_{1}, \ldots, A_{n+1}}$ generalizes both the entrywise product (2) (when $n=1$ ) and the usual matrix product $X_{1} \cdots X_{n}$ (when $\varphi \equiv 1$ ). In the bases of eigenvectors of $A$, the transformation $T_{\varphi}^{A, A, A}$ acts on the pair $(X, Y)$ by

$$
\begin{aligned}
& \left(\left(x_{i j}\right)_{i, j=1}^{d},\left(y_{j k}\right)_{j, k=1}^{d}\right) \\
& \mapsto\left(\sum_{j=1}^{d} \varphi\left(\lambda_{i}, \lambda_{j}, \lambda_{k}\right) x_{i j} y_{j k}\right)_{i, k=1}^{d} .
\end{aligned}
$$

This transformation appears in the representation for the Taylor remainder

$$
\begin{aligned}
& f(A)-f(B)-\left.\sum_{k=1}^{n-1} \frac{1}{k !} \frac{d^{k}}{d t^{k}} f(B+t(A-B))\right|_{t=0} \\
& =T_{f^{[n]}}^{A, B, \ldots, B}(A-B, \ldots, A-B) .
\end{aligned}
$$

Here $f$ is an $n$ times differentiable function whose derivative $f^{(n)}$ is bounded on a segment that contains the spectra of $A$ and $B$, and $f^{[n]}$ is the $n$th order divided difference of $f$, which is defined recursively by

$$
f^{[m+1]}=\left(f^{[m]}\right)^{[1]} .
$$

The relation (7) reduces questions on Taylor approximation and convexity of matrix functions to the analysis of $T_{f^{[n]}}^{A, B, \ldots, B}$.

A multilinear Schur multiplier also calculates the higher-order Fréchet matrix derivative

$$
\begin{aligned}
& \left.\frac{1}{k !} \frac{d^{k}}{d t^{k}} f(B+t(A-B))\right|_{t=0} \\
& =T_{f^{[k]}}^{B, B, \ldots, B}(A-B, \ldots, A-B),
\end{aligned}
$$

where $f$ is $k$ times continuously differentiable. The idea of involving OI in higher-order differentiation of operator functions was introduced by Yu. L. Daletskii and S. G. Krein in 1956, while the best up-to-date results in this area are consequences of modern approaches to OI.

Norm estimates for Schur multipliers. One of the main objectives in the study of multiple operator integrals is finding useful estimates for their norms. While dimension dependent bounds for multilinear Schur multipliers follow directly from their definitions, delicate dimension independent bounds stand on a decades-long development of a comprehensive theory.

The best dimension independent bound is

$$
\begin{gathered}
\left\|T_{\varphi}^{A_{1}, \ldots, A_{n+1}}: \mathcal{S}_{d}^{2} \times \cdots \times \mathcal{S}_{d}^{2} \rightarrow \mathcal{S}_{d}^{2}\right\| \\
=\max _{1 \leq r_{1}, \ldots, r_{n+1} \leq d}\left|\varphi\left(\lambda_{r_{1}}^{(1)}, \ldots, \lambda_{r_{n+1}}^{(n+1)}\right)\right|,
\end{gathered}
$$


where $\mathcal{S}_{d}^{2}$ is the space $\mathcal{B}\left(\ell_{d}^{2}\right)$ equipped with the HilbertSchmidt (Frobenius) norm. The result of (9) is also the simplest one to derive in the collection of dimension independent bounds. Indeed, the inequality $\leq$ in (9) for $n=1$ quickly follows from the entrywise multiplier property of $T_{\varphi}^{A, B}$ and for $n \geq 2$ from the repeated application of Hölder's inequality.

The analog of (9) for $\mathcal{S}_{d}^{p}$, which is the space $\mathcal{B}\left(\ell_{d}^{2}\right)$ equipped with the $p$ th Schatten-von Neumann norm $\|\cdot\|_{p}$, takes the form

$$
\begin{aligned}
& \left\|T_{f^{[n]}}^{A_{1}, \ldots, A_{n+1}}: \mathcal{S}_{d}^{p_{1}} \times \cdots \times \mathcal{S}_{d}^{p_{n}} \rightarrow \mathcal{S}_{d}^{p}\right\| \\
& \quad \leq c_{p_{1}, \ldots, p_{n}}\left\|f^{(n)}\right\|_{\infty} .
\end{aligned}
$$

Here

$$
\begin{aligned}
& 1<p, p_{j}<\infty, j=1, \ldots, n, \\
& \frac{1}{p}=\frac{1}{p_{1}}+\cdots+\frac{1}{p_{n}},
\end{aligned}
$$

$f$ is an $n$ times differentiable function with essentially bounded $f^{(n)}$, and $\|\cdot\|_{\infty}$ denotes the sup norm. A similar bound holds for the seminorm $|\operatorname{Tr}(\cdot)|$, which is smaller than the norm $\|\cdot\|_{1}$, of the multilinear Schur multiplier

$$
\begin{aligned}
& \left|\operatorname{Tr}\left(T_{f[n]}^{A, \ldots, A}\left(X_{1}, \ldots, X_{n}\right)\right)\right| \\
& \leq c_{n}\left\|f^{(n)}\right\|_{\infty}\left\|X_{1}\right\|_{n} \ldots\left\|X_{n}\right\|_{n},
\end{aligned}
$$

where $\operatorname{Tr}$ is the canonical matrix trace and

$$
\|X\|_{n}=\left(\operatorname{Tr}\left(|X|^{n}\right)\right)^{1 / n}
$$

is the $n$th Schatten-von Neumann norm of $X$.

The result of (10) is based on harmonic analysis of Banach spaces with unconditional martingale differences (UMD) and an intricate recursive procedure reducing the order yet preserving the nature of the symbol $f^{[n]}$. The respective approach for $n=1$ was introduced and implemented in [16], its higher-order equivalent in a more technical setting of $n \geq 2$ in [12]. The estimate (11) follows from a suitable generalization of (10) from $f^{[n]}$ to more general symbols given by polynomial integral momenta and from Hölder's inequality.

There is a more universal approach to estimating $T_{f^{[n]}}^{A_{1}, \ldots, A_{n+1}}$, which results in bounds for more general norms but with a coarser dependence on $f^{[n]}$ and a smaller set of admissible functions $f$. Namely,

$$
\begin{aligned}
& \left\|T_{f^{[n]}}^{A_{1}, \ldots, A_{n+1}}: \mathcal{J} \times \cdots \times \mathcal{J} \rightarrow \mathcal{J}\right\| \\
& \leq\left\|f^{[n]}\right\|_{\otimes}
\end{aligned}
$$

where $\mathcal{J}$ is the space $\mathcal{B}\left(\ell_{d}^{2}\right)$ equipped with a unitarily invariant norm, $f$ is a function with smoothness properties stronger than $n$-times but weaker than $(n+1)$-times continuous differentiability, and $\left\|f^{[n]}\right\|_{\otimes}$ is the integral projective tensor product norm of $f^{[n]}$. This norm is generally greater than the norm of $f$ appearing in (10):

$$
\frac{1}{n !}\left\|f^{(n)}\right\|_{\infty}=\left\|f^{[n]}\right\|_{\infty} \leq\left\|f^{[n]}\right\|_{\otimes} .
$$

The inequality (12) is based on a factorization of the function $f^{[n]}\left(\lambda_{1}, \ldots, \lambda_{n+1}\right)$ separating the variables $\lambda_{1}, \ldots$, $\lambda_{n+1}$,

$$
\begin{aligned}
& f^{[n]}\left(\lambda_{1}, \ldots, \lambda_{n+1}\right) \\
& =\int_{\Omega} a_{1}\left(\lambda_{1}, \omega\right) \cdots a_{n+1}\left(\lambda_{n+1}, \omega\right) d \nu(\omega),
\end{aligned}
$$

which was implemented in [11] and [2]. This factorization is in the spirit of the celebrated A. Grothendieck's characterization of bounded linear Schur multipliers on $\mathcal{B}\left(\ell^{2}\right)$. In the next section, we will see alternatives to the bound (12) that reveal dependence on smoothness and decay properties of the function $f$.

From the estimates (9) - (12) and representations (7) and (8) we immediately deduce analogous bounds for derivatives and Taylor remainders of matrix functions.

We note that the Lipschitz-type dimension independent bound

$$
\|f(A)-f(B)\|_{p} \leq c_{p}\|f\|_{\operatorname{Lip}(\mathbb{R})}\|A-B\|_{p},
$$

$1<p<\infty$, which follows for every Lipschitz function $f$ by the same method as (10), does not extend to the trace class norm $(p=1)$ or operator norm. In particular, E. B. Davies showed in 1988 that given $d \in \mathbb{N}$, there exist self-adjoint $A, B \in \mathcal{B}\left(\ell_{2 d}^{2}\right)$ such that

$$
\||A|-|B|\|_{1} \geq \text { const } \log (d)\|A-B\|_{1} .
$$

There are also continuously differentiable functions $f$ with bounded derivative for which the supremum of $\| f(A)-$ $f(B)\left\|_{1} /\right\| A-B \|_{1}$ over distinct self-adjoint $A, B \in \mathcal{B}\left(\ell_{2 d}^{2}\right)$ grows like $\sqrt{\log (d)}$. Higher-order matrix Taylor remainders possess a similar behavior with respect to the trace class norm [15]. Different lower bounds derived in [20] provide a theoretical limitation on the accuracy of matrix Taylor approximations.

Bounds supplied by OI can be used to predict sensitivity of computational problems to small perturbations or rounding errors as well as the accuracy of estimators by sample data. For instance, the norm of a matrix Fréchet derivative calculates a condition number for problems with matrix functions in numerical analysis. For another example, if $A$ is an unknown covariance matrix whose function or functional $f(A)$ is to be estimated and $B$ is a sample covariance matrix, then the bounds for matrix derivatives and Taylor remainders can help to gain insight into the quality of the estimator $f(B)$. 


\section{Operator Integrals on Noncommutative $L^{p}$-spaces}

In the noncommutative analysis involving functions of infinite-dimensional operators with continuous spectra, one works with suitable replacements of the transformations (1) and (6) that still satisfy properties like (3) - (5) and (7) - (12). Nowadays we have a rather comprehensive theory of multilinear operator integration as a cumulative product of many groundbreaking results.

Before proceeding, we briefly recall noncommutative $L^{p}$-spaces where operator integrals are defined. Let $\mathcal{M}$ be a semifinite von Neumann algebra of bounded linear operators acting on a separable Hilbert space $\mathcal{H}$ and let $\tau$ be a normal faithful semifinite trace on $\mathcal{N}$. The noncommutative $L^{p}$-space $L^{p}(\mathcal{M}, \tau), 1 \leq p<\infty$, associated with $(\mathcal{M}, \tau)$ consists of operators affiliated with $\mathcal{M}$ and satisfying

$$
\|X\|_{p}=\left(\tau\left(|X|^{p}\right)\right)^{1 / p}<\infty .
$$

The space $L^{\infty}(\mathcal{M}, \tau)$ is identified with the algebra $\mathcal{M}$.

When $\mathcal{M}$ equals the algebra $\mathcal{B}(\mathcal{H})$ of bounded linear operators on $\mathcal{H}$, the noncommutative $L^{p}$-space coincides with the Schatten-von Neumann ideal $\mathcal{S}^{p}$. The latter consists of compact operators on $\mathcal{H}$ whose sequences of singular values belong to $\ell^{p}$, and the $\mathcal{S}^{p}$-norm is defined to be $\|X\|_{p}=\left(\operatorname{Tr}\left(|X|^{p}\right)\right)^{1 / p}$. The reader unfamiliar with general noncommutative $L^{p}$-spaces can assume the particular case of $\mathcal{S}^{p}$ throughout the note.

Linear case. The first transformation receiving the name "double operator integral" was introduced by Yu. L. Daletskii and S. G. Krein in 1956, following Löwner's utilization of what nowadays is called double operator integration (which was mentioned in the previous section). They proved existence of the derivative $\left.\frac{d}{d t} f(A+t X)\right|_{t=0}$ and estimated its operator norm based on the representation (4), where $T_{f^{[1]}}^{A, A}$ is an iterated Riemann-Stieltjes integral with respect to the spectral family of $A$ and where the class of scalar functions $f$ in their approach is assumed to be more restrictive than turned out to be necessary for existence of the operator derivative. Later in the 1960s, M. S. Birman and M. Z. Solomyak developed several approaches to $T_{\varphi}^{A, B}$ and substantially extended the range of applicability of the double operator integration method, which we sketch below along with more recent approaches.

Let $A, B$ be self-adjoint operators densely defined in $\mathcal{H}$ and let $\varepsilon_{A}, \varepsilon_{B}$ be their spectral measures. The product $\mathcal{E}$ of $\varepsilon_{A}$ and $\varepsilon_{B}$ defined on the rectangular sets $\sigma_{1} \times \sigma_{2}$ of $\mathbb{R}^{2}$ by

$$
\mathcal{E}\left(\sigma_{1} \times \sigma_{2}\right)(X)=\varepsilon_{A}\left(\sigma_{1}\right) X \varepsilon_{B}\left(\sigma_{2}\right)
$$

for every $X$ in the Hilbert space $\mathcal{S}^{2}$ of Hilbert-Schmidt operators on $\mathcal{H}$ is the spectral measure itself. The double operator integral $T_{\varphi}^{A, B}$ on $\mathcal{S}^{2}$ is then defined as the spectral integral

$$
T_{\varphi}^{A, B}(X)=\iint_{\mathbb{R}^{2}} \varphi(\lambda, \mu) d \mathcal{E}(\lambda, \mu)(X),
$$

where $\varphi: \mathbb{R}^{2} \rightarrow \mathbb{C}$ is a bounded Borel function. The transformation $T_{\varphi}^{A, B}$ inherits all the nice properties of a spectral integral, including the inequality

$$
\left\|T_{\varphi}^{A, B}: \mathcal{S}^{2} \rightarrow \mathcal{S}^{2}\right\| \leq\|\varphi\|_{\infty},
$$

which is an analog of (9). M. S. Birman and M. Z. Solomyak showed that the transformation given by (14) satisfies (3) for $A-B \in \mathcal{S}^{2}$ and $f \in \operatorname{Lip}(\mathbb{R})$. The definition (14) and property (15) extend from $\mathcal{S}^{2}$ to a general noncommutative $L^{2}$-space $L^{2}(\mathcal{M}, \tau)$.

If the transformation $T_{\varphi}^{A, B}$ extends from a dense subset $L^{2}(\mathcal{M}, \tau) \cap L^{p}(\mathcal{M}, \tau)$ of the noncommutative $L^{p}$-space $L^{p}(\mathcal{M}, \tau), 1 \leq p<\infty$, to a bounded transformation on $I^{p}(\mathcal{M}, \tau)$, then it inherits properties of a double operator integral, as was realized by B. de Pagter, H. Witvliet, and F. Sukochev in [6]. By the duality $\left(\mathcal{S}^{1}\right)^{*}=\mathcal{B}(\mathcal{H})$, the transformation $T_{\varphi}^{A, B}$ also extends from $\mathcal{S}^{1}$ to $\mathcal{B}(\mathcal{H})$, as was noted by M. S. Birman and M. Z. Solomyak in the 1960 s.

Sufficient conditions for existence of double operator integrals on non-Hilbert spaces and useful estimates for their norms build on analysis of their symbols. One approach relies on existence of a factorization of the symbol $\varphi(\lambda, \mu)$ that separates its variables $\lambda$ and $\mu$. It was first suggested by M. S. Birman and M. Z. Solomyak in 1966 and then generalized by V. V. Peller in 1985 to characterize bounded $T_{\varphi}^{A, B}$ on $\mathcal{B}(\mathcal{H})$. Later, the separation of variables approach to operator integrals was implemented on many symmetrically normed ideals $\mathcal{J}$ of a semifinite von Neumann algebra by N. A. Azamov, A. L. Carey, P. G. Dodds, and F. Sukochev in [2]. These ideals possess a so-called property $(\mathrm{F})$ and include $L^{p}(\mathcal{M}, \tau) \cap \mathcal{M}$ and $L^{p, \infty}(\mathcal{M}, \tau) \cap \mathcal{M}$, $1<p<\infty$. This method is explained below.

Let $\varphi: \mathbb{R}^{2} \rightarrow \mathbb{C}$ admit the representation

$$
\varphi(\lambda, \mu)=\int_{\Omega} a_{1}(\lambda, \omega) a_{2}(\mu, \omega) d \nu(\omega)
$$

with some finite measure space $(\Omega, \nu)$ and bounded Borel functions $a_{i}(\cdot, \cdot): \mathbb{R} \times \Omega \rightarrow \mathbb{C}, i=1,2$, satisfying

$$
\int_{\Omega}\left\|a_{1}(\cdot, \omega)\right\|_{\infty}\left\|a_{2}(\cdot, \omega)\right\|_{\infty} d|v|(\omega)<\infty .
$$

The double operator integral $T_{\varphi}^{A, B}$ is then affixed to the factorization (16) via

$$
\begin{aligned}
& T_{\varphi}^{A, B}(X)(y) \\
& =\int_{\Omega}\left(a_{1}(A, \omega) X a_{2}(B, \omega)\right)(y) d \nu(\omega)
\end{aligned}
$$


for every $y \in \mathcal{H}$. For an ideal $\mathcal{J}$ as above, we obtain

$$
\left\|T_{\varphi}^{A, B}: \mathcal{J} \rightarrow \mathcal{J}\right\| \leq\|\varphi\|_{\otimes}
$$

by the symmetric property of $\mathcal{J}$. Here the norm $\|\cdot\|_{\otimes}$ is defined by

$$
\|\varphi\|_{\otimes}=\inf \int_{\Omega}\left\|a_{1}(\cdot, \omega)\right\|_{\infty}\left\|a_{2}(\cdot, \omega)\right\|_{\infty} d|\nu|(\omega)
$$

with the infimum taken over all possible representations (16). We note that the transformation given by (17) satisfies (3) for $A-B \in \mathcal{B}(\mathcal{H})$ and $f \in \operatorname{Lip}(\mathbb{R})$ with $\varphi=f^{[1]}$ admitting the representation (16). The decomposition (17) is also convenient for passing to the limit with respect to the parameters $A, B$, which was used to prove differentiability results for operator functions.

The existence of the representation (16) for $\varphi=f^{[1]}$ is determined by smoothness and decay properties of the function $f$. Namely, if $f$ belongs to the Besov space $B_{\infty 1}^{1}(\mathbb{R})$, then $f^{[1]}$ satisfies (16) and

$$
\|f\|_{\operatorname{Lip}(\mathbb{R})} \leq\left\|f^{[1]}\right\|_{\otimes} \leq \text { const }\|f\|_{B_{\infty 1}^{1}(\mathbb{R})},
$$

as established by means of the Fourier analysis in V. V. Peller's work in 1985. To see how this analysis works in a nontechnical case, we assume that both $f$ and its Fourier transform $\hat{f}$ are integrable. By the Fourier inversion,

$$
\begin{aligned}
f^{[1]}(\lambda, \mu) & =\frac{f(\lambda)-f(\mu)}{\lambda-\mu} \\
& =\frac{1}{\sqrt{2 \pi}} \frac{1}{\lambda-\mu} \int_{\mathbb{R}}\left(e^{i \lambda t}-e^{i \mu t}\right) \hat{f}(t) d t \\
& =\frac{i}{\sqrt{2 \pi}} \int_{\mathbb{R}}\left(\int_{0}^{t} e^{i(\lambda s+\mu(t-s))} d s\right) \hat{f}(t) d t \\
& =\frac{i}{\sqrt{2 \pi}} \int_{\mathbb{R}^{2}} e^{i \lambda u} e^{i \mu v} \hat{f}(u+v) d u d v .
\end{aligned}
$$

The latter integral is in the form (16), where $\Omega=\mathbb{R}^{2}$, $d \nu(u, v)=\frac{i}{\sqrt{2 \pi}} \hat{f}(u+v) d u d v, a_{1}((u, v), \lambda)=e^{i \lambda u}$, and $a_{2}((u, v), \mu)=e^{i \mu v}$ for $u, v \in \mathbb{R}$. The above calculation and further Fourier analysis induce the bounds

$$
\begin{aligned}
\left\|f^{[1]}\right\|_{\otimes} & \leq\left\|\hat{f}^{\prime}\right\|_{L^{1}(\mathbb{R})} \\
& \leq \sqrt{2}\left(\left\|f^{\prime}\right\|_{L^{2}(\mathbb{R})}+\left\|f^{\prime \prime}\right\|_{L^{2}(\mathbb{R})}\right) .
\end{aligned}
$$

Sharpening dependence on the symbol $f^{[1]}$ for double operator integrals on UMD spaces was crucial for resolution of several open problems. It was implemented by D. Potapov and F. Sukochev in [16] by taking a novel approach and carrying out a different type of harmonic analysis on $f^{[1]}$. The respective transformation $T_{\varphi}^{A, B}$ is constructed by discretizing the spectra of $A, B$, then following the pattern of the finite-dimensional case (1), and finally taking limits to return to the initial operators. More precisely,

$$
\begin{aligned}
& T_{\varphi}^{A, B}(X) \\
& =\lim _{m \rightarrow \infty} \lim _{N \rightarrow \infty} \sum_{l_{1}, l_{2}=-N}^{N} \varphi\left(\frac{l_{1}}{m}, \frac{l_{2}}{m}\right) \varepsilon_{A, l_{1}, m} X \varepsilon_{B, l_{2}, m},
\end{aligned}
$$

where $\varepsilon_{A, l, m}=\varepsilon_{A}\left(\left[\frac{l}{m}, \frac{l+1}{m}\right)\right)$ and $\varepsilon_{A}$ is the spectral measure of $A$. The transformation given by (18) exists for $\varphi=f^{[1]}$, where $f \in \operatorname{Lip}(\mathbb{R})$, and satisfies

$$
\begin{aligned}
& \left\|T_{f^{[1]}}^{A, B}: L^{p}(\mathcal{M}, \tau) \rightarrow L^{p}(\mathcal{M}, \tau)\right\| \\
& \leq c_{p}\|f\|_{\operatorname{Lip}(\mathbb{R})}
\end{aligned}
$$

for $1<p<\infty$. The constant in (19) is known to behave like

$$
c_{p} \sim \frac{p^{2}}{p-1}
$$

as established by M. Caspers, S. Montgomery-Smith, D. Potapov, and F. Sukochev in 2014. The transformations given by (17) and (18) coincide on $\varphi=f^{[1]}$ for a large set of functions $f$, including the space $B_{\infty 1}^{1}(\mathbb{R})$.

There are several proofs of the estimate (19), each one using deep results from harmonic analysis. To demonstrate a few ideas from the original proof, let $\psi$ be a Schwartz function satisfying $\psi(t)=e^{t}$ for $t \in[\log \alpha, \log \beta]$, where $[\alpha, \beta] \subset(0, \infty)$. Then, the Fourier inversion implies that

$$
\begin{aligned}
x & =\psi(t)=\frac{1}{\sqrt{2 \pi}} \int_{\mathbb{R}} \hat{\psi}(s) e^{i s t} d s \\
& =\frac{1}{\sqrt{2 \pi}} \int_{\mathbb{R}} \hat{\psi}(s) x^{i s} d s, \quad x \in[\alpha, \beta] .
\end{aligned}
$$

Hence,

$$
f^{[1]}(\lambda, \mu)=\frac{1}{\sqrt{2 \pi}} \int_{\mathbb{R}} \hat{\psi}(s)|f(\lambda)-f(\mu)|^{i s}|\lambda-\mu|^{-i s} d s
$$

for those $f, \lambda, \mu$ for which $f^{[1]}(\lambda, \mu) \in[\alpha, \beta]$. The latter decomposition of $f^{[1]}$ induces a representation of $T_{f^{[1]}}^{A, A}$ as the integral of compositions of the double operator integrals $T_{\varphi}^{A, A}$ with $\varphi(\lambda, \mu)=|\lambda-\mu|^{-i s}$ and $\varphi(\lambda, \mu)=|f(\lambda)-f(\mu)|^{i s}$. Such transformations are bounded by the MarcinkiewiczMihlin multiplier theory. The respective bounds along with integrability of the moments of $\psi$ ultimately imply the boundedness of $T_{f^{[1]}}^{A, A}$ and estimate (19).

Multilinear case. Attempts to extend linear double operator integrals to the multilinear case and derive analogous useful bounds were driven by applications and made concurrently with development of the double OI theory.

The first practical multiple operator integral construction was implemented on the product of Hilbert spaces in B. S. Pavlov's work in 1969. If $A_{1}, \ldots, A_{n+1}$ are self-adjoint operators densely defined in $\mathcal{H}$ with spectral measures 
$\mathcal{E}_{A_{1}}, \ldots, \mathcal{E}_{A_{n+1}}$, respectively, and $X_{1}, \ldots, X_{n} \in \mathcal{S}^{2}$, then the $\mathcal{S}^{2}$-valued set function $m$ defined on the rectangular sets of $\mathbb{R}^{n+1}$ by

$$
\begin{aligned}
& m\left(\delta_{1} \times \delta_{2} \times \cdots \times \delta_{n+1}\right) \\
& =\varepsilon_{A_{1}}\left(\delta_{1}\right) X_{1} \varepsilon_{A_{2}}\left(\delta_{2}\right) X_{2} \cdots X_{n} \varepsilon_{A_{n+1}}\left(\delta_{n+1}\right)
\end{aligned}
$$

admits extension to a countably additive measure with semivariation bounded by

$$
\|m\| \leq\left\|X_{1}\right\|_{2} \cdots\left\|X_{n}\right\|_{2} .
$$

The multiple operator integral defined by

$$
T_{\varphi}^{A_{1}, \ldots, A_{n+1}}\left(X_{1}, \ldots, X_{n}\right)=\int_{\mathbb{R}^{n+1}} \varphi(\omega) d m(\omega)
$$

for a bounded function $\varphi$ that is measurable with respect to the product of scalar-valued spectral measures of $A_{1}, \ldots, A_{n+1}$ and $X_{1}, \ldots, X_{n} \in \mathcal{S}^{2}$ enjoys the bound

$$
\left\|T_{\varphi}^{A_{1}, \ldots, A_{n+1}}: \mathcal{S}^{2} \times \cdots \times \mathcal{S}^{2} \rightarrow \mathcal{S}^{2}\right\| \leq\|\varphi\|_{\infty} .
$$

The definition (20) does not extend beyond the $\mathcal{S}^{2}$ setting, as confirmed by K. Dykema and A. Skripka in 2009.

There is a more recent approach to the transformation (20) by C. Coine, C. Le Merdy, and F. Sukochev in [5], which adds new technical opportunities. They realized this transformation as a $w^{*}$-continuous contractive map $\varphi \rightarrow T_{\varphi}^{A_{1}, \ldots, A_{n+1}}$ acting on elementary tensors by

$$
\begin{aligned}
& T_{f_{1} \otimes \cdots \otimes f_{n+1}}^{A_{1}, \ldots, A_{n+1}}\left(X_{1}, \ldots, X_{n}\right) \\
& =f_{1}\left(A_{1}\right) X_{1} \cdots X_{n} f_{n+1}\left(A_{n+1}\right) .
\end{aligned}
$$

This realization makes $T_{f_{1} \otimes \cdots, \ldots f_{n+1}}^{A_{1}, ., A_{n+1}}$ amiable to approximation arguments with respect to the parameters $\varphi, A_{1}, \ldots$, $A_{n+1}$. It was recently used to substantially extend results on differentiability of operator functions and obtain the representation (7) for a broad set of of functions.

The double operator integral (17) was extended to the multilinear transformation

$$
T_{\varphi}^{A_{1}, \ldots, A_{n+1}}: \mathcal{B}(\mathcal{H}) \times \cdots \times \mathcal{B}(\mathcal{H}) \rightarrow \mathcal{B}(\mathcal{H})
$$

for $\varphi$ admitting a separation of variables analogous to (16) and to the transformation

$$
T_{\varphi}^{A_{1}, \ldots, A_{n+1}}: \mathcal{J} \times \cdots \times \mathcal{J} \rightarrow \mathcal{J},
$$

where $\mathcal{J}$ is a symmetrically normed ideal with property $(\mathrm{F})$, by V. V. Peller in [11] and by N. A. Azamov, A. L. Carey, P. G. Dodds, and F. Sukochev in [2], respectively. This transformation satisfies the bound

$$
\left\|T_{\varphi}^{A_{1}, \ldots, A_{n+1}}: \mathcal{J} \times \cdots \times \mathcal{J} \rightarrow \mathcal{J}\right\| \leq c_{\mathcal{J}}\|\varphi\|_{\otimes} .
$$

If $f \in B_{\infty 1}^{n}(\mathbb{R})$, then

$$
\left\|f^{[n]}\right\|_{\otimes} \leq \text { const }\|f\|_{B_{\infty 1}^{n}(\mathbb{R})} .
$$

As in the linear case, this OI construction is convenient for passing to the limit with respect to the parameters $A_{1}, \ldots$, $A_{n+1}$.
A transformation $T_{\varphi}^{A_{1}, \ldots, A_{n+1}}$ that captures nice properties of UMD spaces and sharpens dependence on the symbol $\varphi$ was constructed by D. Potapov, A. Skripka, and F. Sukochev in [12] to solve open problems in higher-order noncommutative analysis. This transformation is given by a limit generalizing (18), and it satisfies the bounds (10) and (11) as well as their variants in noncommutative $L^{p}$. spaces. We will discuss the importance of (10) and (11) in the next section.

The estimate (10) is established inductively through an elaborate reduction of the order of $T_{f[n]}^{A_{1}, \ldots, A_{n+1}}$ that preserves major features of its symbol. For instance, a fragment of this reduction for $n=2$ and $\lambda<\mu<\nu$ involves the decomposition

$$
\begin{aligned}
& f^{[2]}(\lambda, \mu, \nu) \\
& =\int_{\mathbb{R}}(\mu-\lambda)^{i s} \varphi_{f^{\prime \prime}}(\lambda, \mu)(\nu-\lambda)^{-i s} \hat{\psi}(s) d s \\
& +\int_{\mathbb{R}}(\nu-\mu)^{i s} \varphi_{f^{\prime \prime}}(\nu, \mu)(\nu-\lambda)^{-i s} \hat{\psi}(s) d s,
\end{aligned}
$$

where $\psi$ is a Schwartz function and

$$
\varphi_{f^{\prime \prime}}(\lambda, \mu)=\int_{0}^{1} t f^{\prime \prime}(\lambda+(\mu-\lambda) t) d t
$$

The decomposition of the symbol $f^{[2]}$ given above leads to the representation of the triple operator integral $T_{f^{[2]}}^{A, A, A}$, subject to the restriction $\lambda<\mu<\nu$, as the integral of compositions of the double operator integrals $T_{\varphi}^{A, A}$ with $\varphi(\lambda, \mu)=(\lambda-\mu)^{ \pm i s}$ (discussed in the previous subsection) and $\varphi(\lambda, \mu)=\varphi_{f^{\prime \prime}}(\lambda, \mu)$, which is similar to $\left(f^{\prime}\right)^{[1]}(\lambda, \mu)$ (also discussed in the previous subsection). The sets like $\lambda<\mu<\nu$ are carved out by triangular truncation operators.

Below we consider several important problems that were solved by methods of multilinear operator integration implemented in different technical setups, each one determined by the intrinsic nature of the problem.

\section{Smoothness of Operator Functions}

In the 1960s, M. G. Krein posed a question on Lipschitz continuity of operator functions that opened a new direction of research in noncommutative analysis. Although existing results of K. Löwner, Yu. L. Daletskii, and S. G. Krein suggested that OI would be a natural tool in questions on operator smoothness, this idea was successfully implemented only in this century after development of a deep and beautiful OI theory.

More precisely, M. G. Krein asked for which functions $f: \mathbb{R} \rightarrow \mathbb{R}$ the respective operator function is Lipschitz in 
$\mathcal{S}^{p}, 1 \leq p<\infty$, that is,

$$
\|f(A)-f(B)\|_{p} \leq c_{f, p}\|A-B\|_{p}
$$

for all self-adjoint $A, B$ such that $A-B \in \mathcal{S}^{p}$. The answer to M. G. Krein's question is twofold. The set of scalar functions giving rise to operator functions that are Lipschitz in the $\mathcal{S}^{p}$-norm, $1<p<\infty$, coincides with $\operatorname{Lip}(\mathbb{R})$, and the bound (13) holds, as established by D. Potapov and F. Sukochev in [16]. However, not every $f \in \operatorname{Lip}(\mathbb{R})$ is Lipschitz with respect to the $\mathcal{S}^{1}$-norm or operator norm, as noted by Yu. B. Farforovskaya in the 1970s. Examples of $A, B$ with $A-B \in \mathcal{S}^{1}$ and continuously differentiable $f \in$ $\operatorname{Lip}(\mathbb{R})$ for which $f(A)-f(B) \notin \mathcal{S}^{1}$ can be constructed by taking direct sums of matrices $A_{d}, B_{d}$ of increasing dimension $d$ for which the quotient $\left\|f\left(A_{d}\right)-f\left(B_{d}\right)\right\|_{1} /\left\|A_{d}-B_{d}\right\|_{1}$ grows logarithmically with $d$, as discussed in the section on finite-dimensional OI.

The set of $\mathcal{S}^{1}$-Lipschitz functions is known to contain those $f \in \operatorname{Lip}(\mathbb{R})$ for which $f^{[1]}$ admits the decomposition (16). It follows from the infinite-dimensional version of (3) and the bound for the respective double operator integral that

$$
\|f(A)-f(B)\|_{1} \leq\left\|f^{[1]}\right\|_{\otimes}\|A-B\|_{1} .
$$

We also have the bound in the weak noncommutative $L^{1}$ quasi-norm

$$
\begin{aligned}
& \|f(A)-f(B)\|_{L^{1, \infty}(\mathcal{M}, \tau)} \\
& \leq \text { const }\|f\|_{\operatorname{Lip}(\mathbb{R})}\|A-B\|_{L^{1}(\mathcal{M}, \tau)}
\end{aligned}
$$

for every $f \in \operatorname{Lip}(\mathbb{R})$, as established by M. Caspers, D. Potapov, F. Sukochev, and D. Zanin in [4].

As distinct from Lipschitzness, the operator functions inherit their Hölder property with $0<\alpha<1$ in the operator norm from scalar functions, according to the result of A. B. Aleksandrov and V. V. Peller [1]. That is,

$$
\|f(A)-f(B)\| \leq c(1-\alpha)^{-1}\|f\|_{\Lambda_{\alpha}(\mathbb{R})}\|A-B\|^{\alpha}
$$

for all self-adjoint $A, B$ with bounded $A-B$ and scalar functions $f$ in the Hölder class $\Lambda_{\alpha}(\mathbb{R})$.

Another aspect of smoothness is differentiability. A function $f$ is $n$ times continuously Fréchet $\mathcal{S}^{p}$. differentiable, $1<p<\infty$, at every bounded self-adjoint operator $A$ on $\mathcal{H}$ if and only if $f \in C^{n}(\mathbb{R})$. This result was obtained by E. Kissin, D. Potapov, V. Shulman, and F. Sukochev in [8] and by C. Le Merdy and A. Skripka in [9] for $n=1$ and $n \geq 2$, respectively. The proof for $n \geq 2$ combines advantages of two approaches to OI introduced in [12] and [5]. The respective $k$ th Fréchet differential is expressed in terms of the multilinear operator integral

$$
\begin{aligned}
& D_{p}^{k} f(A)\left(X_{1}, \ldots, X_{k}\right) \\
& =\sum_{\sigma \in \operatorname{Sym}_{k}} T_{f^{[k]}}^{A, \ldots, A}\left(X_{\sigma(1)}, \ldots, X_{\sigma(k)}\right),
\end{aligned}
$$

where $\operatorname{Sym}_{k}$ is the group of all permutations of the set $\{1, \ldots, k\}$.

When $A$ is unbounded or when the direction of differentiation belongs to another ideal, we do not have a full characterization of Fréchet differentiability. Nonetheless, we have sufficient conditions for existence of the $n$ th-order Fréchet derivatives stated in terms of smoothness and decay properties of $f$ and also a characterization of the $n$ thorder Gâteaux $\mathcal{S}^{p}$-differentiability, $1<p<\infty$. The respective $k$ th-order Gâteaux derivative is given by (8).

Thanks to the representation (7) in the infinite-dimensional case, the estimates for operator integrals apply to operator Taylor remainders

$$
\begin{aligned}
& R_{n, f, B}(A-B) \\
& =f(A)-f(B)-\left.\sum_{k=1}^{n-1} \frac{1}{k !} \frac{d^{k}}{d t^{k}} f(B+t(A-B))\right|_{t=0} .
\end{aligned}
$$

In particular, when the derivatives $\frac{d^{k}}{d t^{k}} f(B+t(A-B))$ are evaluated in the operator norm, we have

$$
\begin{aligned}
& \left\|R_{n, f, B}(A-B)\right\|_{p} \\
& \leq c_{p, n}\left\|f^{(n)}\right\|_{\infty}\|A-B\|_{p n}^{n}, \quad 1<p<\infty,
\end{aligned}
$$

for a self-adjoint bounded $B$ and $f \in C^{n}(\mathbb{R})$. When the operator derivatives are calculated in the $\mathcal{S}^{p}$-norm, $1<$ $p<\infty$, the bound (21) extends to an unbounded $B$ and $n$ times differentiable $f$ with bounded derivatives. We also have

$$
\left\|R_{n, f, B}(A-B)\right\|_{1} \leq c_{n}\|f\|_{B_{\infty 1}^{n}(\mathbb{R})}\|A-B\|_{n}^{n}
$$

for $f \in B_{\infty 1}^{1}(\mathbb{R}) \cap B_{\infty 1}^{n}(\mathbb{R})$. The bound (22) follows from the work of V. V. Peller [11], and it does not extend to all $f \in C^{n}(\mathbb{R})$ by the counterexamples constructed in the work of D. Potapov, A. Skripka, F. Sukochev, and A. Tomskova [15].

\section{Trace Formulas in Mathematical Physics}

Trace formulas relate spectral properties of two operators, where one operator is well understood and the other is viewed as its perturbation whose spectral properties are unknown. This line of research originates from M. G. Krein's seminal 1953 work addressing I. M. Lifshits's findings and questions in physics in the 1940s. Its development and several breakthroughs benefited from the method of multilinear operator integration.

Let $H$ and $V$ be self-adjoint operators, $H$ possibly unbounded. If $V$ belongs to the Schatten-von Neumann ideal $\mathcal{S}^{n}$ for some $n \in \mathbb{N}$, then there exists a unique realvalued function $\eta_{n} \in L^{1}(\mathbb{R})$ depending only on $n, H, V$ such that

$$
\left\|\eta_{n}\right\|_{1} \leq c_{n}\|V\|_{n}^{n}
$$


and

$$
\begin{aligned}
& \operatorname{Tr}\left(f(H+V)-\left.\sum_{k=0}^{n-1} \frac{1}{k !} \frac{d^{k}}{d t^{k}} f(H+t V)\right|_{t=0}\right) \\
& =\int_{\mathbb{R}} f^{(n)}(t) \eta_{n}(t) d t
\end{aligned}
$$

for sufficiently nice $f$. This result was established by M. G. Krein in 1953, L. S. Koplienko in 1984, and D. Potapov, A. Skripka, and F. Sukochev in [12] in the cases $n=1, n=2$, and $n \geq 3$, respectively. The function $\eta_{n}$, called the spectral shift function, plays a fundamental role in perturbation theory, but little is known about properties of $\eta_{n}$ when $n \geq 3$ due to its complexity.

The proof of (23) for $n=2$ utilizes the double operator integrals introduced by M. S. Birman and M. Z. Solomyak to carry out an approximation of $V \in \mathcal{S}^{2}$ by a sequence of finite rank $V_{k}$, for which $\eta_{2}$ can be calculated explicitly in terms of $\eta_{1}$. The trace formula for $n \geq 3$ crucially relies on the estimate (11). Indeed, by the integral representation for the remainder and OI representation for the operator derivative (8),

$$
\begin{aligned}
& f(H+V)-\left.\sum_{k=0}^{n-1} \frac{1}{k !} \frac{d^{k}}{d t^{k}} f(H+t V)\right|_{t=0} \\
& =\frac{1}{(n-1) !} \int_{0}^{1}(1-t)^{n-1} T_{f^{[n]}}^{H_{t}, \ldots, H_{t}}(V, \ldots, V) d t,
\end{aligned}
$$

where $H_{t}=H+t V$. Then the estimate (11) implies existence of the measure $\mu_{n}$ such that the left-hand side of (23) equals $\int_{\mathbb{R}} f^{(n)}(t) d \mu_{n}(t)$ and the total variation of $\mu_{n}$ is bounded by $c_{n}\|V\|_{n}^{n}$. Finally, an approximation argument involving OI and reduction to the lower-order case confirms absolute continuity of $\mu_{n}$ or, equivalently, existence of $\eta_{n}$.

While the condition $V \in \mathcal{S}^{n}$ can hold for perturbations of discrete Schrödinger operators, it does not hold for typical perturbations of differential operators. Instead, when $H$ is a continuous Schrödinger or Dirac operator, its perturbation $V$ can possibly satisfy

$$
(H+V-i I)^{-1}-(H-i I)^{-1} \in \mathcal{S}^{n} .
$$

Analogs of the trace formula (23) were obtained in this extended setting with modified right- and, in some cases, also left-hand sides and the respective $\eta_{n}$ integrable with a weight. These results are based on the theory of spectral shift functions for unitary and contractive operators with perturbations in $\mathcal{S}^{n}$, a change of variables in multiple operator integrals, and creation of summable weights within the OI framework. The condition $(H+V-i I)^{-1}-(H-$ $i I)^{-1} \in \mathcal{S}^{n}$ in the cases $n=1$ and $n=2$ was handled via a reduction to unitaries by M. G. Krein and H. Neidhardt in 1962 and 1988, respectively. The case $n \geq 2$ was treated via a reduction to contractions by D. Potapov, A. Skripka, and F. Sukochev in [14] and $n \geq 3$ via a reduction to unitaries by A. Skripka in [18]. The respective higher-order spectral shift theory for contractions and unitaries builds on OI methods developed in [13] and [18].

The formula (23) extends to more general traces and unsummable perturbations in semifinite von Neumann algebras that arise in noncommutative geometry. In particular, the trace formula with locally integrable $\eta_{n}$ holds for an arbitrary bounded self-adjoint $V$ and unbounded self-adjoint $H$ whose resolvent $(H-i I)^{-1}$ is $\tau$-compact. This property is satisfied, for instance, by differential operators on compact Riemannian manifolds. The trace formulas also hold for perturbations in symmetrically normed ideals equipped with continuous, including singular, traces. They were established for general ideals and $n=1,2$ by K. Dykema and A. Skripka in [7] and for the dual Macaev ideal and $n \geq 3$ by D. Potapov, A. Usachev, F. Sukochev, and D. Zanin in 2015.

\section{Spectral Flow}

The concept of the spectral flow stems from the celebrated work of M. F. Atiyah, V. K. Patodi, and I. M. Singer in the 1970 s, where it was introduced primarily in a topological sense. At the International Congress of Mathematicians in 1974 , I. M. Singer communicated that the spectral flow can be expressed as an integral of 1-form. After J. Phillips introduced an analytic approach to the spectral flow in the context of von Neumann algebras in the 1990s, the implementation of I. M. Singer's suggestion was pursued in the framework of noncommutative geometry. This representation via the integral of a 1-form was confirmed in a general setting without summability restrictions by incorporating double operator integration techniques in the work of N. A. Azamov, A. L. Carey, and F. Sukochev [3].

Let $D_{0}, D_{1}$ be self-adjoint operators affiliated with $\mathcal{M}$ whose resolvents are $\tau$-compact so that $V=D_{0}-D_{1}$ is bounded. If $D_{0}$ and $D_{1}$ are unitarily equivalent, then the spectral flow $\operatorname{sf}\left(D_{0}, D_{1}\right)$ from $D_{0}$ to $D_{1}$ along the path $r \rightarrow D_{0}+r V$ can be calculated by

$$
\operatorname{sf}\left(D_{0}, D_{1}\right)=\frac{1}{\|f\|_{L^{1}(\mathbb{R})}} \int_{0}^{1} \tau\left(V f\left(D_{0}+r V\right)\right) d r
$$

for every nonnegative $f \in C_{c}^{2}(\mathbb{R})$. The expression

$$
\int_{0}^{1} \tau\left(V f\left(D_{0}+r V\right)\right) d r
$$

is the result of integration of the closed exact 1-form $d \theta_{D}^{f}$ given by

$$
\begin{aligned}
& d \theta_{D}^{f}(X) \\
& =\left.\frac{d}{d s}\left(\int_{0}^{1} \tau\left((V+s X) f\left(D_{0}+r V+s r X\right)\right) d r\right)\right|_{s=0} .
\end{aligned}
$$


The above conclusion builds on OI methods for functions of operators with $\tau$-compact resolvents developed by analogy with OI for summable perturbations discussed in the previous section.

When $D_{0}$ and $D_{1}$ are not unitarily equivalent, the representation (24) is modified by the truncated $\eta$-invariants and $\tau$-dimensions of the kernels of $D_{0}$ and $D_{1}$. The spectral flow $\operatorname{sf}\left(D_{0}-\lambda I, D_{1}-\lambda I\right)$ coincides with the spectral shift function $\eta_{1}(\lambda)$ discussed above up to the kernel correction terms.

\section{Quantum Differentiability}

A quantized differential was introduced by A. Connes in the 1980s to replace the differential calculus in noncommutative differential geometry by an operator theoretic notion involving a commutator. The asymptotic behavior of the singular values of the quantized derivative determines the dimension of an infinitesimal in the quantized calculus.

Let $f \in L^{\infty}\left(\mathbb{R}^{d}\right), d \in \mathbb{N}$, and let $M_{f}$ be the operator of pointwise multiplication by $f$. If $D$ is the Dirac operator densely defined in $\mathbb{C}^{\left\lfloor\frac{d}{2}\right\rfloor} \otimes L^{2}\left(\mathbb{R}^{d}\right)$ and $\operatorname{sgn}(D)$ is its sign given by the functional calculus, then the quantized derivative of $f$ is defined to be the commutator

$$
\text { df }:=i\left[\operatorname{sgn}(D), I \otimes M_{f}\right] .
$$

It follows from work of S. Janson and T. H. Wolff in 1982 that $d f \in \mathcal{S}^{d}$ if and only if $f$ is a constant, while the property of the quantized derivative to be in the weak Schattenvon Neumann ideal $\mathcal{S}^{d, \infty}$ is achievable in nontrivial cases. In particular, if $d>1$ and $f \in L^{\infty}\left(\mathbb{R}^{d}\right)$, then $d f \in \mathcal{S}^{d, \infty}$ if and only if $\nabla f \in L^{d}\left(\mathbb{R}^{d}, \mathbb{C}^{d}\right)$. Moreover, there exist constants $c_{d}, C_{d}>0$ such that

$$
c_{d}\|\nabla f\|_{L^{d}\left(\mathbb{R}^{d}, \mathbb{C}^{d}\right)} \leq\|d f\|_{\mathcal{S}^{d, \infty}} \leq C_{d}\|\nabla f\|_{L^{d}\left(\mathbb{R}^{d}, \mathbb{C}^{d}\right)} .
$$

The latter result was proved by S. Lord, E. McDonald, F. Sukochev, and D. Zanin in [10] using the method of double operator integration. The appearance of $\mathrm{OI}$ in this problem is suggested by the formula (5), which extends to the infinite-dimensional case under appropriate assumptions. This variant of (5) gives

$$
\left[D\left(I+D^{2}\right)^{-\frac{1}{2}}, I \otimes M_{f}\right]=T_{g[1]}^{D, D}\left(\left[D, I \otimes M_{f}\right]\right),
$$

where $g(t)=t\left(1+t^{2}\right)^{-1 / 2}$ is the regularized sign function. Results for double operator integrals allow estimation of $T_{g^{[1]}}^{D, D}$ on $\mathcal{S}^{1}$ and on $\mathcal{B}\left(\mathbb{C}^{\left\lfloor\frac{d}{2}\right]} \otimes L^{2}\left(\mathbb{R}^{d}\right)\right)$. Then interpolation transfers this estimate to $T_{g^{[1]}}^{D, D}$ on $\mathcal{S}^{d, \infty}$. Along with a suitable analysis of the commutators $\left[D, I \otimes M_{f}\right]$ and $\left[\operatorname{sgn}(D)-D\left(I+D^{2}\right)^{-\frac{1}{2}}, I \otimes M_{f}\right]$, it ultimately gives the stated upper bound.

\section{Smoothness of Banach Norms}

The study of smoothness properties in function Banach spaces was naturally lifted to a similar investigation in the noncommutative Haagerup $L^{p}$-spaces $L_{\text {Haag }}^{p}(\mathcal{M})$ associated with an arbitrary von Neumann algebra $\mathcal{M}$. This general setting includes the following cases: when $\mathcal{M}$ is semifinite, the space $L_{\text {Haag }}^{p}(\mathcal{M})$ is isometric to the classical noncommutative $L^{p}$-space associated with $\mathcal{M}, 1 \leq p<\infty$; when $\mathcal{M}$ is a type I von Neumann algebra, then $L_{\text {Haag }}^{p}(\mathcal{M})$ contains an isometric copy of the sequence space $\ell^{p}$; when $\mathcal{M}$ is not of type I, $L_{\text {Haag }}^{p}(\mathcal{M})$ contains an isometric copy of the function space $L^{p}(0,1)$.

The first-order Fréchet differentiability of the norm power $\|\cdot\|_{L_{\text {Haag }}^{p}}^{p}, 1<p<\infty$, was essentially established by $\mathrm{H}$. Kosaki in 1984 and its higher-order Fréchet differentiability by D. Potapov, F. Sukochev, A. Tomskova, and D. Zanin in [17]. Namely, we have that $\|\cdot\|_{L_{\text {Haag }}^{p}}^{p}$ is

(i) infinitely many times Taylor-Fréchet differentiable whenever $p$ is an even integer;

(ii) $(p-1)$-times Taylor-Fréchet differentiable whenever $p$ is an odd integer;

(iii) $\lfloor p\rfloor$-times Taylor-Fréchet differentiable whenever $p$ is not an integer.

More specifically, for self-adjoint $A, X \in L_{\text {Haag }}^{p}(\mathcal{M})$,

$$
\begin{aligned}
& \|A+X\|_{L_{\text {Haag }}^{p}}^{p} \\
& =\|A\|_{L_{\text {Haag }}^{p}}^{p}+\sum_{k=1}^{[p-1]} \delta_{k}^{A}(X, \ldots, X)+\mathcal{O}\left(\|X\|_{L_{\text {Haag }}^{p}}^{p}\right),
\end{aligned}
$$

where $\delta_{k}^{A}$ is a symmetric $k$-linear bounded functional on $L_{\text {Haag }}^{p}(\mathcal{M}) \times \cdots \times L_{\text {Haag }}^{p}(\mathcal{M})$. When $p$ is even, $\mathcal{O}\left(\|X\|_{L_{\text {Haag }}^{p}}^{p}\right)=$ $\delta_{p}^{A}(X, \ldots, X)$. The differentials $\delta_{k}^{A}$ are given in terms of multilinear operator integrals.

Since $\|X\|_{L_{\text {Haag }}^{p}}^{p}=\psi\left(|X|^{p}\right)$, where $\psi$ is a normalized trace on the weak noncommutative $L^{1}$-space $L^{1, \infty}(\mathcal{N})$ associated with a certain semifinite crossed product von Neumann algebra $\mathcal{N}$, the analysis of smoothness properties of $\|\cdot\|_{L_{\text {Haag }}^{p}}^{p}$ reduces to the analysis of the operator function arising from the scalar function $f(t)=|t|^{p}$. The latter analysis is fulfilled by means of multilinear operator integration developed on weak noncommutative $L^{p}$-spaces $L^{p, \infty}(\mathcal{N})$, which contain $L_{\text {Haag }}^{p}(\mathcal{M})$ as a closed subspace. One of the features specific to this setting is involvement of Calderón-type operators in derivation of Hölder-type estimates for OI.

\section{Closing Remarks}

In this brief journey to operator integration we considered only the case of self-adjoint operators $A_{1}, \ldots, A_{n+1}$. Multilinear operator integration has also been developed in the 
cases when $A_{1}, \ldots, A_{n+1}$ are unitary, contractive, dissipative, or unbounded normal operators. The theory has also been extended to noncommutative weak $L^{p}$-spaces and, in the linear case, to general Banach spaces. The details can be found in [19].

While we demonstrated usefulness of operator integration on selected representative applications, the OI method has more to offer. Further general results based on this method along with their variants designed for specific models of mathematical physics and noncommutative geometry can be found in [19], thematic surveys, and recent and upcoming articles.

\section{References}

[1] Aleksandrov AB, Peller VV. Operator Hölder-Zygmund functions, Adv. Math., no. 3 (224):910-966, 2010. MR2628799

[2] Azamov NA, Carey AL, Dodds PG, Sukochev FA. Operator integrals, spectral shift, and spectral flow, Canad. J. Math., no. 2 (61):241-263, 2009, DOI 10.4153/CJM-2009-012-0 MR2504014

[3] Azamov NA, Carey AL, Sukochev FA. The spectral shift function and spectral flow, Comm. Math. Phys., no. 1 (276):51-91, 2007, DOI 10.1007/s00220-007-03299. MR2342288

[4] Caspers M, Potapov D, Sukochev F, Zanin D. Weak type commutator and Lipschitz estimates: resolution of the Nazarov-Peller conjecture, Amer. J. Math., no. 3 (141):593610, 2019, DOI 10.1353/ajm.2019.0019 MR3956516

[5] Coine C, Le Merdy C, Sukochev F. When do triple operator integrals take value in the trace class?, arXiv 1706.01662 https://arxiv.org/abs/1706 .01662

[6] de Pagter B, Witvliet H, Sukochev FA. Double operator integrals, J. Funct. Anal., no. 1 (192):52-111, 2002, DOI 10.1006/jfan.2001.3898. MR1918492

[7 Dykema K, Skripka A. Perturbation formulas for traces on normed ideals, Comm. Math. Phys., no. 3 (325):1107-1138, 2014. MR3152748

[8] Kissin E, Potapov D, Shulman V, Sukochev F. Operator smoothness in Schatten norms for functions of several variables: Lipschitz conditions, differentiability and unbounded derivations, Proc. Lond. Math. Soc. (3), no. 4 (105):661-702, 2012, DOI 10.1112/plms/pds014. MR2989800

[9] Le Merdy C, Skripka A. Higher order differentiability of operator functions in Schatten norms, J. Inst. Math. Jussieu. https://doi.org/10.1017/S1474748019000033

[10] Lord S, McDonald E, Sukochev F, Zanin D. Quantum differentiability of essentially bounded functions on Euclidean space, J. Funct. Anal., no. 7 (273):2353-2387, 2017, DOI 10.1016/j.jfa.2017.06.020 MR3677828

[11] Peller VV. Multiple operator integrals and higher operator derivatives, J. Funct. Anal., no. 2 (233):515-544, 2006, DOI 10.1016/j.jfa.2005.09.003. MR2214586

[12] Potapov D, Skripka A, Sukochev F. Spectral shift function of higher order, Invent. Math., no. 3 (193):501-538, 2013, DOI 10.1007/s00222-012-0431-2. MR3091975
[13] Potapov D, Skripka A, Sukochev F. Higher-order spectral shift for contractions, Proc. Lond. Math. Soc. (3), no. 2 (108):327-349, 2014. MR3166355

[14] Potapov D, Skripka A, Sukochev F. Trace formulas for resolvent comparable operators, Adv. Math. (272):630-651, 2015, DOI 10.1016/j.aim.2014.12.016 MR3303244

[15] Potapov D, Skripka A, Sukochev F, Tomskova A. Multilinear Schur multipliers and Schatten properties of operator Taylor remainders, Adv. Math. (320):1063-1098, 2017, DOI 10.1016/j.aim.2017.09.012 MR3709129

[16] Potapov D, Sukochev F. Operator-Lipschitz functions in Schatten-von Neumann classes, Acta Math., no. 2 (207):375-389, 2011. MR2892613

[17] Potapov D, Sukochev F, Tomskova A, Zanin D. Fréchet differentiability of the norm of $L_{p}$-spaces associated with arbitrary von Neumann algebras, Trans. Amer. Math. Soc., no. 11 (371):7493-7532, 2019, DOI 10.1090/tran/7215 MR3955526

[18] Skripka A. Estimates and trace formulas for unitary and resolvent comparable perturbations, Adv. Math. (311):481509, 2017, DOI 10.1016/j.aim.2017.02.026 MR3628221

[19] Skripka A, Tomskova A. Multilinear Operator Integrals: Theory and Applications, Lecture Notes in Mathematics, vol. 2250, 2019.

[20] Skripka A, Zinchenko M. Stability and uniqueness properties of Taylor approximations of matrix functions, Linear Aleebra Appl. (582):218-236, 2019, DOI 10.1016/j.laa.2019.07.037 MR3992426

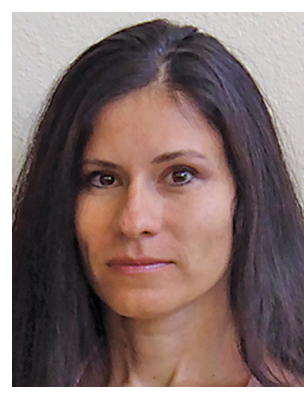

Anna Skripka

Credits

Opener image is courtesy of Getty.

Author photo is courtesy of the author. 